\title{
Multiple Victims: The Result of Caring Patients in Vegetative State
}

\author{
Fateme Goudarzi ${ }^{1}$; Heidarali Abedi ${ }^{2, *}$; Kourosh Zarea ${ }^{1}$; Fazlollah Ahmadi ${ }^{3}$ \\ ${ }^{1}$ Department of Nursing, Nursing and Midwifery School, Ahvaz Jundishapur University of Medical Sciences, Ahvaz, IR Iran \\ Department of Nursing, Nursing and Midwifery School, Ahvaz Jundishapur University of Medical Scien
2 Faculty of Nursing and Midwifery, Isfahan (Khorasgan) Branch, Islamic Azad University, Isfahan, IR Iran \\ ${ }^{3}$ Department of Nursing, Faculty of Medical Sciences, Tarbiat Modares University, Tehran, IR Iran \\ ${ }^{*}$ Corresponding Author: Heidarali Abedi, Faculty of Nursing and Midwifery, Isfahan (Khorasgan) Branch, Islamic Azad University, Isfahan, IR Iran. Tel: +98-3135354058, Fax: \\ +98-3135354065, E-mail: habedi@khuisf.ac.ir \\ Received: September 12, 2014; Revised: September 29, 2014; Accepted: October 22, 2014
}

\begin{abstract}
Background: Having a patient in a vegetative state in the family is a complicated and stressful experience. Caring for such patients with complete disability at home is very challenging.

Objectives: The present study aimed to explore the outcomes of caring for patients in a vegetative state for families and caregivers at home.

PatientsandMethods:In this qualitative study,16 vegetativepatients' caregivers were selected through purposive sampling. Unstructured interviews and observations were used for data gathering. Data collection was continued until saturation of data and emergence of the main themes. Data analysis was performed by the content analysis method.

Results: The analysis of the gathered data led to three themes: "lost main caregiver", "affected caring partner" and "affected family". Each theme had some subthemes and subcategories.

Conclusions: The three emerged themes in this study showed that all the family members of vegetative patients, depending on their responsibilities, were affected by physical, mental, social and economic issues.
\end{abstract}

Keywords: Caregivers; Persistent Vegetative State (PVS); Vegetative State; Nursing

\section{Background}

Vegetative State (VS) is one of the Disorders of Consciousness (DOC) (1); a condition which originates from the coma state (1-3), and occurs through full or relative return of the brain stem $(1,4)$, thalamic functions (4), wakefulness capacity and opening of the eyes (5). Therefore, patients in VS are awake, yet they fail to attain awareness about themselves and the environment, to have purposeful behavioral responses to stimuli, and to comprehend language and to communicate (1-3). The term 'vegetative' as explained in the oxford dictionary, refers to "an organic body capable of growth and evolution while unable to think and feel" (6). Regardless of the challenges in naming this term, it refers to the lack of capability and need of vegetative patients for thorough but dignified and humanized care (7).

The prognosis of VS after some months is weak and is improbable after a year $(3,8)$. However, the advancements in cardiopulmonary resuscitation techniques, surgery and critical care for patients with severe brain injury have saved the lives of many vegetative patients, and consequently have brought forth longer lives for these patients (6, 9-11). Saout reported the prevalence of vegetative patients with a wide range of 56 to 140 per million (12). On the other hand, these patients will survive as long as they are provided with artificial hydration and nutrition $(13,14)$.
The increasing frequency and long survival of such patients intensify caring difficulties. Due to the special condition of these patients, they need continuous medical and social interferences and widespread nursing care (15-18). The person who provides care, their skills and the location at which care is provided are all important for patient outcome. For example, in Taiwan such patients are cared for in hospital-based special care units or in nursing homes (19). In some countries including Iran, due to the long period of illness, these patients are discharged from the hospital after their conditions are stabilized, therefore, their care continues at home (20). The family thus plays a vital role in such patients' lives and care $(16,18,20,21)$.

Having a vegetative patient in the family is a complicated (13) and stressful experience (22) accompanied by various issues of concern such as ambiguity in patient's condition and uncertainty about the length of illness (13), high costs (23), and the stress due to lack of therapeutic effect despite the costs (24).

Among the family members, the main caregivers are more influenced because of the allocated amount of time and their emotional involvement in caring (25). In a study by Chiambretto et al. vegetative patients' caregivers declared that the full-time care made them isolated 
in such a way that they had no time to meet friends, visit places of interest, participate in outdoor activities, study and watch television (26). The findings of the study of Moretta et al. also showed higher levels of psychological distress in vegetative patients' caregivers (27). Caring for such patients in the family is, therefore, very challenging and bears various negative outcomes. However, most studies conducted in this regard were limited to quantitative assessment of caregivers from the perspective of mental health $(10,11,13,16,21,25,27-29)$, and other effects on caregivers and the other members of the family have not been focused upon. Moreover, the conducted studies were quantitative, while description and exploration of unknown or less known phenomena is only possible through true qualitative studies $(30,31)$.

\section{Objectives}

The aim of the present study was to explore the affects of caring for vegetative patients on families and caregivers at home.

\section{Patients and Methods}

\subsection{Design}

In this study, content analysis was used for gathering and analyzing the data with an aim to abstract and describe the phenomenon, and analyze outcomes with concepts and categories which describe the phenomenon (32).

\subsection{Setting and Participants}

This study was conducted during 2013 and 2014. The key participants were family caregivers of vegetative patients who had at least one month of caring experience of their patients at home and were purposefully entered into the study. They were selected by maximum variation sampling based on age, gender, caring duration, relationship with the patient, marital status and education. Due to the lack of caring and supporting centers for such patients and lack of diagnosis in hospital records, the researcher found them by querying the hospital staff, especially those who provide at home care. Therefore, the environment of the study was wherever a trace of these patients could be found. Sampling was thus conducted in some provinces of Iran (Khuzestan, Lorestan and Tehran). The participants included 16 individuals who cared for vegetative patients at home. Thirteen were family members that cared for the patients, and three were professional caregivers. Most of the family caregivers were females and immediate members of the family (including mother, wife, son or daughter, sister or brother), and only in one case the caregiver was the brother's wife. The average length of caring was 20.35 months (from 1.5 to 108 months). Professional caregivers were mostly males, and often nurse assistants who had the average experience of 9.67 years. Other characteristics of the participants are presented in Table 1.
Table 1. Demographic Characteristics of the Participants $(n=$ 16) ${ }^{\mathrm{a}}$

\begin{tabular}{lc}
\hline Variable & Values \\
\hline Family Caregivers & $30.77 \pm 7.18$ \\
\hline Age, y & $10(76.92)$ \\
\hline Gender & $3(23.08)$ \\
\hline Female & $20.35 \pm 28.50$ \\
\hline Male & \\
\hline Caring Duration, m & $33 \pm 6.08$ \\
\hline Professional Caregivers & $1(33.33)$ \\
\hline Age, y & $2(66.66)$ \\
\hline Gender & $9.67 \pm 8.33$ \\
\hline Female & \\
\hline Male & \\
\hline Working Experience, y & \\
\hline a Data are presented as Mean \pm Standard Deviation (SD) or No. (\%).
\end{tabular}

\subsection{Data Collection}

To gather data, unstructured and face-to-face interviews were performed. Due to the comfort of the participants, the interviews were conducted at home for family caregivers, and in researcher's office at the university for professional caregivers. The average time of the interviews was 55.76 minutes (from 15 to 122). In interviewing family caregivers, one general question was asked to explain their experience during care, and in some cases they were asked to explain a day of care for such patients. The professional caregivers were also asked to explain their experience of caring for such patients. Then, during interviews, some prop questions were asked based on the participants' statements to deepen the interviews. The interviews were recorded with the participants' permission. Data gathering and analysis were synchronized. After identifying themes and reaching data saturation, the interviews were stopped. Saturation is the point at which all concepts are well defined (33) and new data isn't attained and all levels of codes are completed (34). The data obtained from the two observations (a professional caregiver trained a family caregiver and took notes about caring for a patient in a vegetative state by a family caregiver at home) were also analyzed. MAXQDA10 software was used for data analysis.

\subsection{Data Analysis}

Data analysis was done based on the steps proposed by Granheim and Lundman. Analysis units included 16 interviews and two observations. The text of the interviews and notes about the observations were transcribed verbatim and read several times to gain a sense of the entire situation. Then, meaningful units related to parts and sections of data were summarized. The meaningful units were condensed and abstracted, and were then given 
Goudarzi F et al.

appropriate codes and labels. The obtained codes were constantly compared based on their similarities and differences. Similar codes were, therefore, categorized. The categories are a descriptive level of the manifest content. Determination of the relationship between categories was done through extraction of themes, and themes were interpreted signifying the data's latent content (35).

\subsection{Trustworthiness}

To ensure validity and reliability of data in this study, Lincoln and Guba's four criteria including credibility, dependability, conformability and transferability were taken into consideration (36). To increase the reliability, the researcher was engaged with the data for more than one year. The transcripts of the interviews, codes and categories were scrutinized by colleagues experienced in qualitative studies (other members of the research team). If there were disagreements, the researchers discussed and clarified the cases to reach a consensus. Through the member check process, the codified transcripts of the interviews were given to three participants, and the codes were verified. To provide auditing, the process and procedure of the study was carefully recorded and reported.

\subsection{Ethical Considerations}

The present study was approved by the Research council affiliated with the Ahvaz Jundishapur University of Medical Sciences (NO.ajums. REC. 1392, 202). Necessary information concerning the aim and purpose of the study was given to the participants, and written informed consents were obtained from them before entering the study. They were ascertained about the confidentiality of their information and anonymity, and they could leave the study at any stage.

\section{Results}

The analysis of the gathered data led to three themes: "lost main caregiver", "affected caring partner" and "affected family". Subthemes and subcategories related to these themes are presented in Table 2.

\subsection{Lost Main Caregiver}

The statements of the participants indicated that, because of the "hard caring" which imposed "various injuries", the vegetative patients' main caregivers in the family experienced the most physical and mental pressures compared with the other members of the family. One of the participants said: "I experienced the highest physical and mental pressure” (P12).

\subsubsection{Hard Caring}

"Hard caring" had three subcategories: "high needs of the patient", "comprehensiveness of care" and "time-consuming caring" which made caring difficult.
Table 2. Themes and Subthemes Related to Multiple Victims in Caring for Patients in a Vegetative State

\begin{tabular}{|l}
\hline Themes \\
\hline Lost main caregiver \\
\hline Hard caring \\
\hline High needs of the patient \\
Comprehensiveness of care \\
Time-consuming caring \\
\hline Various injuries \\
\hline Physical injuries \\
\hline Disordered daily activities \\
\hline Standstill in job and studies \\
\hline Isolation \\
\hline Mental distress \\
\hline Fatigue \\
\hline Affected caring partner \\
\hline Physical injuries \\
\hline Standstill in job and studies \\
\hline Affected family \\
\hline Other affected members \\
\hline Family mental deterioration \\
\hline Family financial erosion \\
\hline Expensive caring \\
\hline High expenses \\
\hline Financial deterioration \\
\hline
\end{tabular}

\subsubsection{High Needs of the Patient}

All the participants declared that patients in a vegetative state are unable, totally dependent and need comprehensive care. Therefore, they compared such patients with new-born babies or flowers and plants to describe their dependency. Due to complete incapability, they needed high levels of care. In this regard, one of the participants said: "this patient needs a lot care, when I feed him, it takes an hour. Can you imagine how much it takes to medicate and suction him, to clean his mouth and other things?" (P5).

\subsubsection{Comprehensiveness of Care}

Family caregivers offered comprehensive care including "hygienic-supportive care" and "various imposed specialized care" for their patients. The main caregivers, in offering hygienic-supportive care to patients in a vegetative state paid close attention to hygienic considerations (including replacement of bedding and clothing, oral health considerations, bathing, caring and cleaning eyes), caring of pressure areas (including change of position and massage) and stimulating the senses of hearing and vision. Some of the statements of the participants were as followed: "we even brush his teeth and use dental 
floss to keep them from getting decayed" (P15). "To prevent the development of bedsores, it's our duty to stay awake late at midnight to change his position, we change his position every two hours" (P6).

What clarified the comprehensiveness of care was the "various imposed specialized cares" including nursing considerations related to drug and fluid therapy, intravenous line, tracheostomy, suction of pulmonary secretions, sore care, urination, defecation and nutrition, checking vital signs, and even obtaining medical specimens conducted by family caregivers. Of course, at first, the caregivers did not have the technical knowledge, but they learned as they tried to meet the needs of their patients. Food and drug gavage, suction of pulmonary secretions, and checking vital signs were among less dangerous technical cares, which all caregivers did for their patients based on training. In one of the observational notes, the researcher witnessed that a "family caregiver after connecting the gavage syringe to the PEG (Percutaneous endoscopic gastrostomy), she filled it with milk, situated it $30 \mathrm{~cm}$ above, and opened the clamp to let it enter the stomach with appropriate speed. Before the milk in the syringe was completely finished, she clamped it to add drugs and let it enter the stomach. At the end, some water was given and after clamping, the syringe was separated and washed." (observational note related to P6). One of the participants said: "whenever we see he has shortness of breath or is coughing or has discharge, we perform the suction for him" (P7).

Tracheostomy care, replacing dressing, injecting drugs, even embedding urinary tube and nasogastric tube were among specialized cares with high risk which were performed by family caregivers. Some of the participants said: "We made the sore bleeding by gauze and irrigation serum to remove the dead tissues and dressed it up" (P1). "I learned nasogastric tube insertion and was replacing it every ten days" (P3).

\subsubsection{Time-Consuming Caring}

The frequency and repetition of the above-mentioned care requires a long duration of time. Most of the caregivers expressed that such time consuming care stopped them from paying attention to themselves. One of the participants said: "caring for such patients is very difficult, I had to look after them from morning to night, and I even had no time to rest" (P14).

\subsubsection{Various Injuries}

The main caregiver in the family experienced many issues such as "physical injuries", "disordered daily activities", "standstill in job and studies", "isolation", "mental distress" and "fatigue" due to the long-time spent caring. Herniated disk and knee and arm pain were among the main caregivers' "physical injuries". One participant said: "scapular pain has been added to my backache and knee pain because I mostly use this arm to move, feed and exer- cise him. I don't want his body to become stiff" (P8).

The main caregivers described "disordered daily activities" such as disorders in sleeping, nutrition, hygiene, sexual intercourse and paying attention to their health. Participants in this regard expressed that "they're next to him day and night; they never sleep" (P9, Professional caregiver). "We never go anywhere for recreation, we can't go, if we want to, we won't feel comfortable" (P5).

Family members engagement in care in many cases led to "standstill in job and studies". In this regard, participants said: "I worked as a notary, and I couldn't hold this job because of my dad"(P2). "He worked for oil companies, but he doesn't anymore" (P9, Professional caregiver).

"Domestic imprisonment" because of the full-time nature of care and "limited relationship" with surroundings and the society led to "isolation" of these caregivers. Here is what some of the participants said: "during these three years he was kept at home, I went out of the house only five times, I've been imprisoned at home" (P1). "I've thoroughly cut off my relationship with other people; I used to be in touch with the society, but from the time my dad got ill, I'm only in contact with him" (P2).

"Mental distress" in the form of depression and aggression and "fatigue" were among problems that all participants emphasized upon. Their expressions included: "sometimes, I feel depressed, and I weep to calm down" (P12). "I've witnessed how her temper was affected. Sometimes she becomes aggressive to his sister and mother. She has no control over his behavior" (P13, Professional caregiver). "I feel fatigue because I do almost all his caring tasks" (P5).

\subsection{Affected Caring Partner}

In caring for patients in a vegetative state apart from the main caregiver, who had the major responsibilities, another member of the family cooperated directly with the main caregiver in providing care. This person was the caring partner. What main caregivers and their caring partners said showed that the caring partner was not invulnerable against problems and injuries, either. "Physical injuries" such as pain in the back and hands were among issues, which affected the caring partners. One of participants said: "my mom, my sister and I look after him, we change his position and take him to the bathroom, the three of us currently have constant backache" (P16). "Standstill in job and studies" was another issue faced by the caring partners: "I gave up my job, I decided to go to university, but I couldn't” (P10)

\subsection{Affected Family}

In the process of caring for patients in a vegetative state, not only were the main caregiver and the caring partner affected, but also other members of the family were influenced as well, in one way or another. This theme includes three sub-themes: "other affected members", "family mental deterioration" and "family financial erosion". 
Goudarzi F et al.

\subsubsection{Other Affected Members of the Family}

In families with patients in a vegetative state, even members not directly involved in caring, experienced problems such as mental distress and even standstill in their job or studies. The participants stated: "apart from me, my dad suffered the most stress" (P12). "My brother was a taxi driver, who sold his car to pay for treatment expenses of our dad and now he is jobless" (P5).

\subsubsection{Family Mental Deterioration}

"Family mental deterioration" compared with before the patients' illness was among challenges that most of the participants declared. One participant stated: "Our family was happy but they are not anymore, all are upset, depressed and nervous" (P4).

\subsubsection{Family Financial Erosion}

"Family financial erosion" was another problem that all families with a vegetative patient were confronted with. This erosion was because of "expensive caring" of such patients due to "expensive necessities" including various consumable and non-consumable necessities, "expensive nutrition" and "expensive procedures".

"Diversity and plurality of consumption necessities" for patients in a vegetative state such as diapers, types of catheters (urinary and gastrointestinal tube and nelaton), serum, gauze and drugs were among the reasons why such care was expensive. The participants stated: "there are some supplies such as nelaton, adhesive paper, gauze and his drugs which are frequently used" (P5). "Diverse and expensive non-consumable products" such as floating mat, suction machine, oxygen capsule, wheelchair, pulse oximeter and even disinfection devices led to other costs, which made care of these patients expensive. The subjects claimed that: "bed, floating mat and suction machine are the necessary things they need at the beginning; you can imagine how much they cost?" (P13, professional caregiver). "Expensive procedures" such as visiting a doctor, home nurse, and occupational and physical therapist were other factors contributing to the expenses: "every 10 or 15 days, a doctor visits him at home, the visit costs 40,000 Tomans" (P2). "Every time a nurse comes to our house, it costs 10 to 15 thousand Tomans for procedures such as gastric or urinary tube insertion, drug injection, or dressing his wounds" (P16). "Expensive nutrition" also imposes high expenses on families. The participants notified that: "the patient's nutrition is very expensive because their food must be various including vitamins, different types of meat and natural juices" (P4). Therefore, due to the "high expenses", all the families experienced "financial deterioration" in the long term. One of the caregivers said: "those families with such patients have high costs, such costs paralyze the family" (P10).

\section{Discussion}

The first theme, "lost main caregiver", showed that the main caregivers are affected by "various injuries" because of the "hard caring". "Hard Caring" was the result of the variety and high volume of caring needs. In the present study, various types of care such as considerations related to hygienic needs, pressure areas, sensory stimulation, drugs and fluid therapy, I.V. line (intravenous line), tracheostomy, suction of pulmonary secretions, sore care, urination, defecation, nutrition, checking for vital signs and obtaining samples from patients were performed by family caregivers. In a study conducted by Lavrijsen et al. similar daily care for patients in a vegetative state was reported which was, of course, performed by professional caregivers in nursing homes (8). In the present study, based on what the participants said, performing the above-mentioned daily care made them thoroughly engaged in caring for the patients and consequently they had no time for themselves. While, in the study of Leonardi et al. it was reported that participants spent more than three hours for their relatives in a vegetative state (21). The difference between full-time care in the present study and three-hours of care in the study of Leonardi et al. could be due to the fact that their care was performed in special centers and by the personnel of the centers, while, in our study, family caregivers performed various types of care and monitored the patient at all times.

Due to the mentioned "hard caring", the main caregivers in this study experienced "various injuries" such as "physical injuries", "disordered daily activities", "standstill in job and studies", "isolation", "mental distress" and "fatigue". Therefore, the burden of caring must be considered as an interconnected multidimensional issue with physical, mental and social dimensions (21).

Backache was a "physical injury" experienced by most family caregivers. Therefore, caring for absolutely incapable and dependent patients in a vegetative state leads to heavy physical injuries for the caregivers (28). "Disordered daily activities", especially sleep and recreation disorders resulting from full-time care were among family caregivers' experiences. Alongside the findings of the present study, Leonardi et al. also discovered that most caregivers emphasized on the decrease in leisure time, especially in outdoor activities, social involvement and leisure activities such as jogging and cycling (21). In a study by Moretta et al. most of the participants temporarily or permanently abandoned their previous activities due to the reduced time they allocated to themselves and their hobbies, which imposed a high burden and negative effects on their quality of life (27). "Standstill in job" in the form of giving up or losing job was another issue described by the participants in the present study. Due to spending long durations of time for daily care or even in some cases for transferring the patients to cities with specialized units, the main caregivers gave up their jobs (28). In the study of Leonardi et al. over one-third of the samples were forced to temporarily or permanently abandon their jobs, which placed them in inappropriate economic conditions due to low wages or lack of wages (21). "Isola- 
tion" in the form of "limited relationships" and "domestic imprisonment" was another experience of the family caregivers in the present study. Most of the samples in the study of Leonardi et al. stated that their visiting of friends decreased, therefore limited social relationships and decreased outdoor hobbies were characteristics of their daily activities (21). Caregivers in this study considered their "mental distress" as depression. The findings of some studies concerning mental effects of caring for patients in a vegetative were consistent with the present study and showed some signs of depression among caregivers $(16,21,28)$. Other studies in this regard showed other mental effects such as prolonged grief disorder $(13,28)$, PTSD (post-traumatic stress disorder) (28), and even severe mental disorders among caregivers (27). Therefore, mental distress was a frequent problem among caregivers. "Fatigue" was another issue faced by caregivers in the present study. A previous study indicated that daily hours spent providing care was an important predictive factor for fatigue so that if daily caring exceeded three to four hours, caregivers' burnout would be more (16), however fatigue was probably more severe in our study since care was provided on a full-time basis.

The second theme, "affected caring partner" showed that despite the less responsibility and caring burden and fewer allocated hours to care, the caring partners were not invulnerable against injuries and had physical injuries and in some cases had to give up their jobs or studies. It is important to mention that when a family member is sick, incapable or injured, all the family members are affected (37) and experience high anxiety and distress (24).

The third theme, "affected family", showed that all the family members of the patient in a vegetative state were affected. Living with a patient in a vegetative state is a stressful experience for all family members (24) and imposes various burdens on physical and socio-psychological aspects, and had direct and indirect financial expenses (16), which mostly affect functional capabilities of the families (37).

"Mental deterioration" of the family was among the experiences of the participants explained as sadness, depression and aggression. Due to the stress related to this difficult condition, the families of such patients experienced a wide range of feelings and anxieties such as isolation, fear of the future, and fear of sin, fear of insufficiency, sadness, fatigue, excessive protection, aggression and anger (24). Another important issue faced by the patients' families was "financial erosion". Crispi and Crisci in a study aimed at showing the attitudes and feelings of the patients' relatives, found that the participants repeatedly mentioned financial problems and explained their lives as poor and miserable (23). This indicates that financial issues are important to these families and can affect their attitudes. As Gustavsson stated, having a disabled family member with a long-term inability such as DOC can impose multiple burdens on the families and caregivers due to its direct and indirect expenses (29). The direct expenses, based on the participants' experiences, included consumption and non-consumption necessities, expensive nutrition and caring procedures such as visiting doctors, nurses and physical and occupational therapists. Patients' disability and unemployed of caregivers decreases the family income and consequently imposes indirect expenses. Based on the data from Italy's census bureau, the annual income of families with vegetative patients was one-third of typical families, and such families considered themselves to belong to economically lower classes of the society (21). However, financial conditions plays a vital role in meeting the high needs of such patients (38).

Three themes "lost main caregiver", "affected caring partner", and "affected family" emerged in this study and it was shown that all family members of patients in a vegetative state, depending on their responsibilities, developed some degree of physical, mental, social and economic injuries. It is not boastful to say that caring for patients in a vegetative state victimizes their family because the entire family becomes paralyzed in different aspects. The findings showed that caring for patients in a vegetative state at home was accompanied with many expenses; however, such expenses can be used in other parts of public health and health system through correct planning and policy-making. In doing so, discharge training must be more emphasized and follow-up sessions should take place after discharge. Establishing care or support centers can be helpful for such patients and their families. Families and caregivers of such patients must be supported in different aspects such as financial, physical and mental throughout the caring process (29).

\subsection{Limitations}

Similar to other qualitative studies, the findings of the present study should cautiously be generalized. However, due to the similarities of caring context of patients in a vegetative state in Iran, the findings are applicable all over Iran. Moreover, establishing care or support centers can help families and solve the problem of accessibility to such patients, which was among the limitations of the present study.

\section{Acknowledgements}

This paper was part of a dissertation of a nursing PhD candidate (Fateme Goudarzi). The researchers appreciate the Vice Chancellor for Research Affairs of Ahvaz Jundishapur University of Medical Sciences for financially supporting this study (Grant NO.U-92176). We also thankfully acknowledge the patients' families and caregivers who participated in this research.

\section{Authors' Contributions}

Fateme Goudarzi developed the study design, conducted the data collection and analysis, and drafted the manuscript. Heidarali Abedi was the supervisor. Fazlol- 
lah Ahmadi and Kourosh Zarea were the consultants who supervised the data gathering process, data analysis and coding, and revised the manuscript. All authors approved the final manuscript.

\section{Funding/Support}

This study was supported by the Ahvaz Jundishapur University of Medical Sciences (Grant NO. U-92176).

\section{References}

1. Vincent JL, Abraham E, Moore FA, Kochanek PM, Fink MP. Textbook of critical care. 6 edPhiladelphia: Elsevier Saunders; 2011.

2. Goldman L, Schafer AI. Goldman's Cecil medicine. 24 edUSA: Working together to grow libraries in developing countries; 2010

3. Longo D, Fauci A, Kasper D, Hauser S. Harrison's Principles of Internal Medicine .18 edNew York: McGraw-Hill Professional; 2011.

4. Medical aspects of the persistent vegetative state (1). The MultiSociety Task Force on PVS. N Engl J Med.1994;330(21):1499-508.

5. Latronico N. Anaesthesia, pain, intensive care and emergency A. P. I. C. E.Milan: Springer; 2006.

6. Jennett B. Thirty years of the vegetative state: clinical, ethical and legal problems. Prog Brain Res. 2005;150:537-43.

7. Buchini S, Quattrin R, Zampieron A. Valuing dignity in patients in a vegetative state on an intensive rehabilitation ward: improvement project. J Nurs Manag. 2014;22(2):140-50.

8. Lavrijsen J, van den Bosch H, Koopmans R, van Weel C, Froeling P. Events and decision-making in the long-term care of Dutch nursing home patients in a vegetative state. Brain Inj. 2005;19(1):67-75.

9. Beis JM, Seyer JL, Brugerolle B, Le Chapelain L, Thisse MO, Mainard $\mathrm{D}$, et al. Care protocol for persistent vegetative states (PVS) and minimally conscious state (MSC) in Lorraine: retrospective study over an 18-year period. Ann Phys Rehabil Med. 2009;52(5):374-81.

10. Chiambretto P, Moroni L, Guarnerio C, Bertolotti G, Prigerson HG. Prolonged grief and depression in caregivers of patients in vegetative state. Brain Inj. 2010;24(4):581-8.

11. Cipolletta S, Gius E, Bastianelli A. How the burden of caring for a patient in a vegetative state changes in relation to different coping strategies. Brain Inj. 2014;28(1):92-6.

12. Saout V, Ombredane MP, Mouillie JM, Marteau C, Mathe JF, Richard I. Patients in a permanent vegetative state or minimally conscious state in the Maine-et-Loire county of France: A cross-sectional, descriptive study. Ann Phys Rehabil Med. 2010;53(2):96-104.

13. Elvira de la Morena MJ, Cruzado JA. Caregivers of patients with disorders of consciousness: coping and prolonged grief. Acta Neurol Scand. 2013;127(6):413-8.

14. Position of the American Academy of Neurology on certain aspects of the care and management of the persistent vegetative state patient. Adopted by the Executive Board, American Academy of Neurology, April 21, 1988, Cincinnati, Ohio. Neurology. 1989;39(1):125-6.

15. Donis J, Kraftner B. The prevalence of patients in a vegetative state and minimally conscious state in nursing homes in Austria. Brain Inj. 2011;25(11):1101-7.

16. Giovannetti AM, Leonardi M, Pagani M, Sattin D, Raggi A. Burden of caregivers of patients in Vegetative state and minimally conscious state. Acta Neurol Scand. 2013;127(1):10-8.

17. Kitzinger J, Kitzinger C. The 'window of opportunity' for death after severe brain injury: family experiences. Sociol Health Illn. 2013;35(7):1095-112.
18. Schulz R, O'Brien A, Czaja S, Ory M, Norris R, Martire LM, et al Dementia caregiver intervention research: in search of clinical significance. Gerontologist. 2002;42(5):589-602.

19. Lin LC, Hsieh PC, Wu SC. Prevalence and associated factors of pneumonia in patients with vegetative state in Taiwan. J Clin Nurs. 2008;17(7):861-8.

20. Black JM, Hawks JH, Keene AM. Medical-surgical nursing: Clinical management for positive outcomes. 8 edSt. Louis: WB Saunders Co; 2009.

21. Leonardi M, Giovannetti AM, Pagani M, Raggi A, Sattin D, National Consortium F, et al. Burden and needs of 487 caregivers of patients in vegetative state and in minimally conscious state: results from a national study. Brain Inj. 2012;26(10):1201-10.

22. Lewis SL, Heitkemper MM, Dirksen SR, O'Brien PG, Bucher L. Medical-surgical nursing (assessment \& management of clinical problem). 7 edSt. Louis: Mosby Elsevier; 2007.

23. Crispi F, Crisci C. Patients in persistent vegetative state ... and what of their relatives? Nurs Ethics. 2000;7(6):533-5.

24. Li YH, Xu ZP. Psychological crisis intervention for the family members of patients in a vegetative state. Clinics (Sao Paulo). 2012;67(4):341-5.

25. Pagani M, Giovannetti AM, Covelli V, Sattin D, Raggi A, Leonardi M. Physical and mental health, anxiety and depressive symptoms in caregivers of patients in vegetative state and minimally conscious state. Clin Psychol Psychother. 2014;21(5):420-6.

26. Chiambretto P, Rossi Ferrario S, Zotti AM. Patients in a persistent vegetative state: caregiver attitudes and reactions. Acta Neurol Scand. 2001;104(6):364-8.

27. Moretta P, Estraneo A, De Lucia L, Cardinale V, Loreto V, Trojano L. A study of the psychological distress in family caregivers of patients with prolonged disorders of consciousness during inhospital rehabilitation. Clin Rehabil. 2014;28(7):717-25.

28. Guarnerio C, Prunas A, Della Fontana I, Chiambretto P. Prevalence and comorbidity of prolonged grief disorder in a sample of caregivers of patients in a vegetative state. Psychiatr Q. 2012;83(1):65-73.

29. Pagani M, Giovannetti AM, Covelli V, Sattin D, Leonardi M. Caregiving for patients in vegetative and minimally conscious states: perceived burden as a mediator in caregivers' expression of needs and symptoms of depression and anxiety. J Clin Psychol Med Settings. 2014;21(3):214-22.

30. Munhall PL. Nursing research (A qualitative perspective). 4 edBoston: Jones and Bartlett; 2007.

31. Speziale HS, Streubert HJ, Carpenter DR. Qualitative research in nursing: Advancing the humanistic imperative.Philadelphia: Lippincott Williams \& Wilkins; 2011.

32. Elo $\mathrm{S}$, Kyngas $\mathrm{H}$. The qualitative content analysis process. J Adv Nurs. 2008;62(1):107-15.

33. Corbin J, Strauss A. Basics of qualitative research: Techniques and procedures for developing grounded theory.London: Sage publications; 2008.

34. Moule P, Goodman M. Nursing research (An introduction).Los Angeles: SAGE; 2009.

35. Graneheim UH, Lundman B. Qualitative content analysis in nursing research: concepts, procedures and measures to achieve trustworthiness. Nurse Educ Today. 2004;24(2):105-12.

36. Lincoln Y, Guba E. Naturalistic Inquiry.Newbury Park: CA: SAGE Publication; 1985

37. Smeltzer SC, Bare BG, Hinkle JL, Cheever KH. Brunner \& Suddarth's textbook of Medical-Surgical Nursing.Philadelphia: Wolters Kluwer/Lippincott Williams \& Wilkins; 2010.

38. Cohen-Almagor R. Language and reality at the end of life. J Law Med Ethics. 2000;28(3):267-78. 